\title{
THE STUDY OF SOCIO-MENTAL STATUS OF MOTHER AND INFANT GROWTH IN UNDER 6 MONTHS CHILDREN IN AHVAZ
}

\author{
P. Shahri, S. Azarnoosh, M.H. Haghighizadeh \\ Ahvaz Jundishapur University of Medical Sciences, Ahvaz, Iran
}

Introduction: Physical growth is a reliable index for evaluation of health status in children especially in the first two years of life. This study was conducted to evaluate the relationship of maternal socio- mental factors and growth of under 6 months children out in western health centers in Ahvaz.

Methods: Current study was a cross-sectional and analytical study which was carried out in western health centers in Ahvaz 2008-2009. Subjects were 200 under 6 months children who referring to health centers and were selected through random sampling Data were collected using a standard questionnaire GHQ-28 to evaluate mothers` mental health status and a checklist was used for recording children growth indexes like weight, height, head and chest circumferences and mother's demographic information. T -Test, Spearman correlation Statistical analysis were conducted by using Statistical SPSS 16. $(\mathrm{P}<0.05)$.

Results: Using Spearman correlation test there was a significant association among the weight gain and head circumference in children and maternal age and economical status $(\mathrm{P}<0 / 01)$, also a significant relationship was seen among education and mental status of mother with weight gain and head circumference $(\mathrm{P}<0 / 05)$. Although there was not a relationship among growth of children and maternal job and her physical health.

Conclusion: Regarding to findings, maternal characteristics like age, education and mental status have an important relation with infant growth. So these indicate the need for implementing of strategies for recognition of mother's risk factors and specific infant care programs concerning the importance of maternal characteristics. 